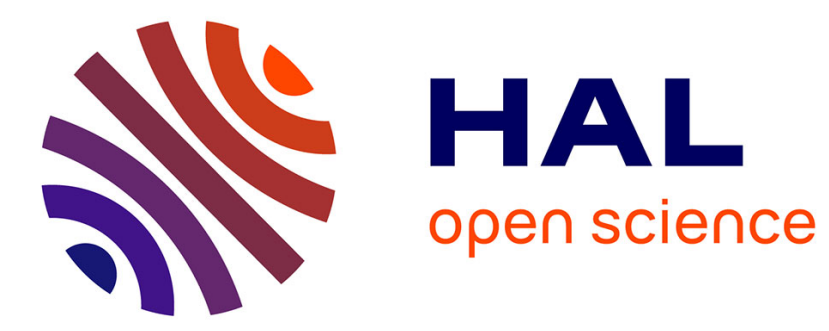

\title{
Evolutionary processes transpiring in the stages of lithopanspermia
}

\author{
Ian von Hegner
}

\section{To cite this version:}

Ian von Hegner. Evolutionary processes transpiring in the stages of lithopanspermia. 2020. hal$02548882 \mathrm{v} 2$

\section{HAL Id: hal-02548882 \\ https://hal.science/hal-02548882v2}

Preprint submitted on 5 Aug 2020

HAL is a multi-disciplinary open access archive for the deposit and dissemination of scientific research documents, whether they are published or not. The documents may come from teaching and research institutions in France or abroad, or from public or private research centers.
L'archive ouverte pluridisciplinaire HAL, est destinée au dépôt et à la diffusion de documents scientifiques de niveau recherche, publiés ou non, émanant des établissements d'enseignement et de recherche français ou étrangers, des laboratoires publics ou privés. 


\title{
Evolutionary processes transpiring in the stages of lithopanspermia
}

\author{
Ian von Hegner \\ Aarhus University
}

\begin{abstract}
Lithopanspermia is a theory proposing a natural exchange of organisms between solar system bodies as a result of asteroidal or cometary impactors. Research has examined not only the physics of the stages themselves but also the survival probabilities for life in each stage. However, although life is the primary factor of interest in lithopanspermia, this life is mainly treated as a passive cargo. Life, however, does not merely passively receive an onslaught of stress from surroundings; instead, it reacts. Thus, planetary ejection, interplanetary transport, and planetary entry are only the first three factors in the equation. The other factors are the quality, quantity, and evolutionary strategy of the transported organisms. Thus, a reduction in organism quantity in stage 1 might increase organism quality towards a second stress challenge in stage 3. Thus, robustness towards a stressor might in fact be higher in the bacterial population surviving after transport in stage 3 than at the beginning in stage 1 . Therefore, the stages of lithopanspermia can themselves facilitate evolutionary processes that enhance the ability of the collected organisms to survive stresses such as pressure and heat shock. Thus, the multiple abiotic pressures that the population encounters through the three stages can potentially lead to very robust bacteria with survival capacities considerably higher than might otherwise be expected. This analysis details an outcome that is possible but probably rare. However, in addition to lithopanspermia, spacecraft mediated panspermia may also exist. The analogous stages in a spacecraft would result in a greater likelihood of increasing the stress tolerance of hitchhiking organisms. Furthermore, missions seeking life elsewhere will frequently be sent to places where the possibility of life as we know it is assumed to exist. Thus, we not only can transport terrestrial organisms to places where they are potentially more likely to survive but also may increase their invasive potential along the way. This analysis highlights further requirements that planetary protection protocols must implement and also provides a framework for analyses of ecological scenarios regarding the transmission of life, natural or artificial, between worlds in a solar system.
\end{abstract}

Key words: astrobiology, adaption, meteorites, planetary protection.

\section{Introduction}

The possibility of meteorites as carriers of organisms was entertained by E. de Montlivault (1821), when he suggested that meteorites could be carriers of life to Earth, whereas Hermann Richter (1865) proposed a hypothesis detailing how some meteors could partially penetrate the Earth's atmosphere at such an angle that they could potentially collect airborne organisms along the way, before re-entering space with the organisms. Since then, lithopanspermia, the theory proposing natural exchange of organisms between solar system bodies, owing to asteroidal or cometary impactors, has become a well-established field of research in astrobiology [Nicholson, 2009].

Scientific studies have focused on the following three distinct stages: (1) planetary ejection, in which organisms must survive ejection from a world; (2) interplanetary transit, in which organisms must survive transmission through space; and (3) planetary entry, in which organisms must survive entry from space into a world. These stages encompass multidisciplinary research seeking to clarify whether organisms can survive each of these stages and estimate the overall chances of survival when organisms are transferred between solar system bodies [von Hegner, 2020].

One of the early criticisms of lithopanspermia was based on doubt as to whether material could even be exchanged between solar system bodies. However, since its proposal, evidence has indicated that among the over 62,000 meteorites found on Earth [The Meteoritical Society, 2020], 142 are Martian in origin [Irwing, 2020], thus eliminating any doubts as to the exchange in material between planets. The opposite situation, in which terrestrial material arrives on Mars, also appears conceivable.

Importantly, the key mechanism underlining lithopanspermia is not restricted to the past but instead remains active in the solar system [Beech et al., 2018]. An example is the 2011 arrival of the Tissint 
meteorite [Brennecka et al., 2014], which demonstrated that meteorites have continued to be transmitted between solar system bodies to the present day.

Whether the organisms transported within the ejecta can survive all three stages is being actively investigated. Although only a few organisms have been studied, new research data continue to be generated. The current consensus appears to be that organisms may indeed survive all three stages of lithopanspermia [Beech et al., 2018]. The notion that life can survive transmission to another world and that life has actually been transmitted to another world are, of course, two different situations. Whether life has ever been transmitted between worlds in this solar system is not yet known. Although the probability of successful transfer of life between worlds appears modest, the possibility nevertheless appears to exist.

Therefore, if the conjecture is granted that life can indeed be transmitted safely from, for example, Earth to Mars or the moons of Jupiter or Saturn, or vice versa, then it is a topic within not only planetary science but also biological science, thus necessitating a framework combining the physical dynamics of lithopanspermia and the dynamics of evolutionary processes. Where there is life, there is evolution, and it follows from evolutionary theory that species have the potential to modify their stress tolerance and thus their invasiveness potential. Therefore, in this article, I will seek to clarify the potential implications of this prediction in the three stages of lithopanspermia.

\section{Discussion}

Darwinian evolution can be defined as a competition for survival among individual organisms within any given species - it can also be defined as a competition among genes within gene pools. Furthermore, organisms not only compete against each other but also adapt to changing environments. Thus, populations that experience environmental change gradually adapt to acquire one or more traits. Consequently, optimal trait values change continuously, and natural selection results in the gradual evolution of more fit phenotypes, thus removing suboptimal forms that hitherto were well adapted to the environment [Simons, 2011]. Thus, in evolutionary theory, robustness - that is, a capacity to survive adverse environmental conditions caused by, e.g., heat stress or cold stress in a given species - can be predicted to be a trait that the species gradually acquires in response to a harsh environment [Lenz et al., 2018].

Research into the possibility of lithopanspermia has focused on the following three distinct stages: (1) planetary ejection, (2) interplanetary transport, and (3) planetary entry. The research focus has been on the ejection rates, the dynamic transfer and impact physics, and the subjection of the organisms to stresses such as intense radiation, desiccation due to exposure to the vacuum of space etc. Thus, although life is the primary factor of interest in lithopanspermia, this life remains treated as a passive cargo, and the research focus is on aspects such as how many organisms survive a given pressure shock, and the rates of metabolic function degradation or cellular repair, etc. all which are vital to the understanding of lithopanspermia.

However, life does not passively receive an onslaught of stress from the surroundings. If life actively survives this onslaught, it responds. Thus, planetary ejection, interplanetary transport, and planetary entry are just the first three relevant factors in the equation. The other factors are the quality, quantity, and evolutionary strategy of the transported organisms [von Hegner, 2020]. The three stages of lithopanspermia represent, to put it mildly, a changed environment for organisms, and the environmental stressors encountered therein will thus provoke evolutionary responses.

A hypothesis can be proposed in which robustness or stress tolerance can also be acquired during an invasion process involving transport of members of one species from one region to another over a certain period of time [Lenz et al., 2018]. Here, mortality during transport due to stress increases the mean tolerance to the same type of stress in the group of transported organisms due to an increase in the frequency of robust genotypes [Sakai et al., 2001]. That is, the mortality rates are lower among the survivors of a preceding stress event than among the non-stressed organisms of the same species. Thus, population robustness towards a stressor is higher at the end of transport than at the beginning [Gavan et al., 2016; Lenz et al., 2018]. In this article, the following assumptions will be made:

(i) The mean robustness of organisms towards a stressor is higher at the end of interplanetary transport than at the beginning. This is a prevalent biological effect among bacteria.

(ii) Meteorites bring living organisms to other worlds. 
(iii) The transported organisms can remain active in stage 2.

Given the general toughness and versatility of bacteria compared with other terrestrial species, and the fact that they are the dominant species in this world, existing in all available niches, their likelihood of being picked up and surviving the journey is highest; therefore, this article focuses on bacteria. In section 3, I will discuss the evolutionary processes occurring in the three stages, which I will call stage 1 and stage 3, and stage 2, the stages of lithopanspermia and invasiveness. Then in section 4, I will discuss implications for planetary protection protocols.

\section{Adaptation}

The three stages of lithopanspermia have been suggested to be able to transport a bacterial (or archaeal) population between solar system bodies. This transmission exposes the encapsulated organisms to stressful abiotic conditions. Thus, because organisms adapt to a changing environment and have the potential to modify their stress tolerance, the question arises as to what potential processes can occur in the three stages of lithopanspermia.

\section{Stage 1 and Stage 3}

During stage 1, the stressful abiotic conditions experienced by the bacterial population are pressure shock and heat shock.

The few surviving bacteria able to cope with stage 1 would also be expected to have an opportunity to survive stage 3, because robustness towards stresses, such as heat stress or cold stress, in a species can be an inherent trait that has gradually evolved in response to a harsh environment. Thus, if the first stage eliminates all the bacteria unable to cope with the pressure and heat shock, the surviving bacteria possessing these genetically pre-adapted robust genotypes would also be expected to potentially be able to survive the pressure and heat shock in stage 3.

This is the classic scenario. Here, life is merely a passive cargo being transported from the donator world to the acceptor world by a meteorite. However, the situation may be more complex. Interestingly, bacteria can actually achieve greater robustness than offered in this classic scenario.

When an asteroidal or cometary impactor hits and ejects material from a world, stage 1 of lithopanspermia involves a change from a temperate to a briefly elevated temperature. Hence, during temperate transportation of bacteria that starts and ends with a briefly increased temperature event, all bacteria inside the ejected material will experience temperature changes.

Thus, during stage 1, temperatures will be reached that constitute heat shock for the bacterial population, and this heat shock may lead to a decrease in quantity, that is, to partial mortality among the collected bacterial population, thereby selecting for bacterial genotypes with the greatest tolerance towards heat shock. Hence, the quality of the bacteria should increase afterwards; that is, the frequency of heat shock tolerant genotypes in the bacterial population, should increase during stage 2. Interestingly, however, the stress tolerance towards the second stress event of the same type can be higher than would otherwise be expected on the basis of robust genotypes alone. This aspect can be illustrated by a thought experiment, as shown in Figure 1.

In this scenario, bacterial population A undergoes the traditional stages of lithopanspermia. This population of bacteria experiences pressure and heat stress twice, in stage 1 and after a recovery phase in stage 2, during which the pressure fades and the heat decreases; the population again experiences pressure and heat stress in stage 3 .

In contrast, hypothetical bacterial population B of the same species experiences a gentle launch through, e.g., a space elevator [Artsutanov, 1960], rather than the violent ejection in stage 1. This other population of bacteria experiences pressure and heat stress only once, in stage 3 . These two bacterial populations spend the same amount of time in each meteorite in stage 2 and experience the same conditions.

The stress that bacterial population A experiences through stage 1 can be expected to cause major mortality in the population. Thus, it is now the case that the surviving individuals experience a second stress event of the same type in stage 3, and their survival is then compared with the robustness of bacterial 
population $\mathrm{B}$, which underwent a gentle launch in the space elevator and thus did not experience these elevated pressure and heat shocks until stage 3 .

The stress tolerance in survivors of population A can be expected to be enhanced by the previous pressure and heat stress, in comparison to the stress tolerance of population B. Hence, the robustness of bacterial population A towards these pressure and heat stressors should be higher at the end of the interplanetary transmission, in stage 3 , than at the beginning, at stage 1 .

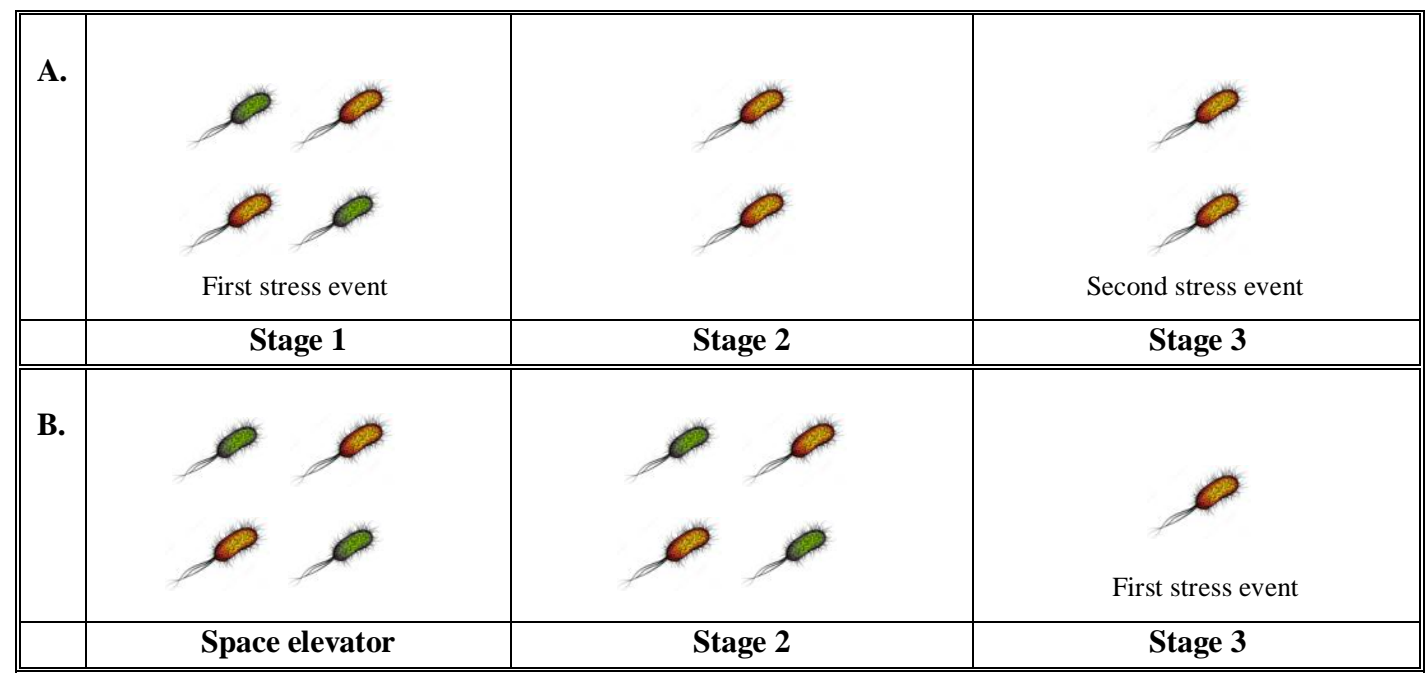

Figure 1. Thought experiment comparing the mortality rates of bacterial populations A and B in stage 3 . A. The bacterial population undergoing the traditional stages of lithopanspermia. This first group of bacteria experiences pressure and heat shock twice, in stage 1 and after a recovery phase in stage 2, in stage 3 as well. B. The hypothetical bacterial control population that is gently launched from the world by a hypothetical space elevator without pressure and heat shock. The group experiences pressure and heat shock only once, in stage 3. Credits: bacteria adapted from Mirumur, 2011.

Thus, in stage 1, the asteroidal or cometary impactor will initially initiate a natural selection of pressure and heat tolerant bacteria, and the reduction in quantity, that is, the stress-induced mortality among them, will increase the quality of the surviving bacterial population, that is, the average robustness towards pressure and heat stress, in stage 3, as a consequence of the bacteria having previously experienced these stressors.

Experiments supporting this prediction have been performed. Gavan et al. (2016) have studied the stress resistance of a small fraction of severely heat stressed E. coli. The surviving E. coli were initially only slightly more sensitive to a second heat treatment but were more resistant to a second shock against another stressor, such as high hydrostatic pressure shock, than were unstressed control E. coli. However, as the resuscitation phase continued, the initial high hydrostatic pressure resistance of the initially sublethally injured E. coli was observed to fade, whereas their heat resistance increased and eventually surpassed the initial heat resistance of the unstressed E. coli [Gavan et al., 2016].

Lenz et al. (2018) have performed a study mimicking the heat stress experienced by mussels during a transoceanic voyage. In this study, groups of individual mussels, which often hitchhike in hull fouling or ballast water tanks in ships, were exposed to thermal stress that caused $60-83 \%$ mortality in the groups. The surviving mussels were later exposed to a second heat stress event. In three of the studied mussel species, no clear difference in resistance to heat stress was observed between groups of individual mussels that survived a previous heat event and mussels that were not previously heat stressed. However, two mussel species demonstrated an increased resistance to heat stress when they had survived a previous heat stress event [Lenz et al., 2018].

Experiments testing the survival of organisms during pressure shocks simulating stage 1 have also been performed. Horneck et al. (2008) have conducted shock recovery experiments within a pressure range deduced from Martian meteorites; these authors, among others, have studied pressure shock of the endolithic cyanobacterium Chroococcidiopsis. The number of survivors in Chroococcidiopsis sp. decreased with 
increasing shock pressures, surviving shock pressures up to $10 \mathrm{GPa}$ (survival rate nearly $10^{-3}$ ) before reaching survival below the threshold of detection. Hence, at pressures greater than $10 \mathrm{GPa}$, all identified cells were lysed [Horneck et al., 2008].

In an initial consideration, the number of survivors in stage 1 of these experiments was not necessarily due to random survival among the cyanobacteria, i.e., a statistical fluke; instead, the experiments selected for individual genotypes with a pre-adapted tolerance to pressure shock, thus allowing the bacteria with the greatest genetically adapted robustness to survive longer. However, the increased tolerance towards a second stress event of the same type is apparently not due to preadapted mutants, because Gavan et al. (2016) have found that most heat stressed E. coli survivors are not heat resistant mutants displaying a steadily increased robustness towards heat stress. Hence, the heat shock has instead been suggested to either trigger a translation independent response or to be conferred as a yet poorly understood physiological state upon the surviving bacteria that mitigates the effects of the second stress event [Gavan et al., 2016].

As for heat shock, a hypothesis can here be made in which pressure shock tolerance can also be acquired. Hence, after a recovery phase under normal conditions, the surviving bacteria should show greater robustness to a second pressure shock event than a control group experiencing this pressure shock event only once.

Thus, in the stages of lithopanspermia, a reduction in quantity among the bacterial population can be expected to increase the quality in the surviving population by not only increasing the frequency of robust genotypes but also conferring a physiological state upon the surviving bacteria that mitigates the effects of the second stress event.

In this scenario, the bacterial population is not merely a passive cargo transported by a meteorite. Life itself is active and dynamic; it responds to the environment and can thus affect the outcome of the transmission itself as well as a successful deposition in another world. Thus, the first stage can in itself be considered a facilitator of a process enhancing the ability of the collected organisms to survive stage 3 .

\section{Stage 2}

The discussed scenario requires the meteorite to protect the bacteria in the transmission in stage 2 so that they have a recovery phase and thus actively maintain their most basic functions.

In most cases organisms, organisms might survive only the transmission between two solar system bodies in the form of bacterial endospores, which have long been studied with regard to space conditions. These spores are the hardiest terrestrial life form known and are capable of enduring extreme conditions in their dormant state that otherwise damage or are lethal to other known forms of life [Nicholson et al., 2000]. Calculations by Mileikowsky et al. (2000) have predicted that a fraction of a Bacillus subtilis spore population $\left(10^{-6}\right)$ would in fact survive cosmic radiation in space for approximately $1 \mathrm{Ma}$ if protected by $1 \mathrm{~m}$ of meteorite material (if the rock is assumed to initially harbour approximately $10^{8}$ spores $/ \mathrm{g}$ ) and $25 \mathrm{Ma}$ if protected by 2 to $3 \mathrm{~m}$ of meteorite material. Thus, the natural transfer of microorganisms such as Deinococcus radiodurans and Bacillus sp. has been concluded to be not only possible but highly probable in Mars-Earth interplanetary travel.

However, if organisms make the transmission in a dormant state, then they are unable to actively maintain their most basic functions. That scenario is analogous to the few bacteria surviving stage 1 , which can be expected to potentially survive stage 3 , because they already possess the inherent trait robustness that evolved in response to a harsh environment in the donator world. However, the bacteria would then not achieve an increase in the mean tolerance to the same type of stress in stage 3 . Thus, they must be active in stage 2 for this increase to be in effect.

However, although time is required, the travel times between two solar system bodies such as e.g. Mars and Earth are, from a biological point of view, often quite long, thereby decreasing the possibility of organisms remaining active in stage 2 for long periods, owing to a lack of resources and the stress of space. Most Martian meteorites are estimated to have spent from approximately $\sim 0.6$ to 15 million years in space. For example, the Martian meteorite Nakhla, which fell on 28 June, 1911, in northern Egypt, had a transmission time in space of approximately $~ 10.75$ million years [Nyquist et al., 2001], and the Tissint 
meteorite is estimated to have been ejected between 700,000 and 574 million years ago [Brennecka et al., 2014].

However, on the basis of calculations, shorter travel times may be possible, such that some meteorites potentially reach transit trajectories rapidly enough to allow for interplanetary travel lasting months to several years between Mars and Earth [Melosh, 1988]. However, notably, the greater the pressure shocks at launch and impact, the lower the probability of organism survival.

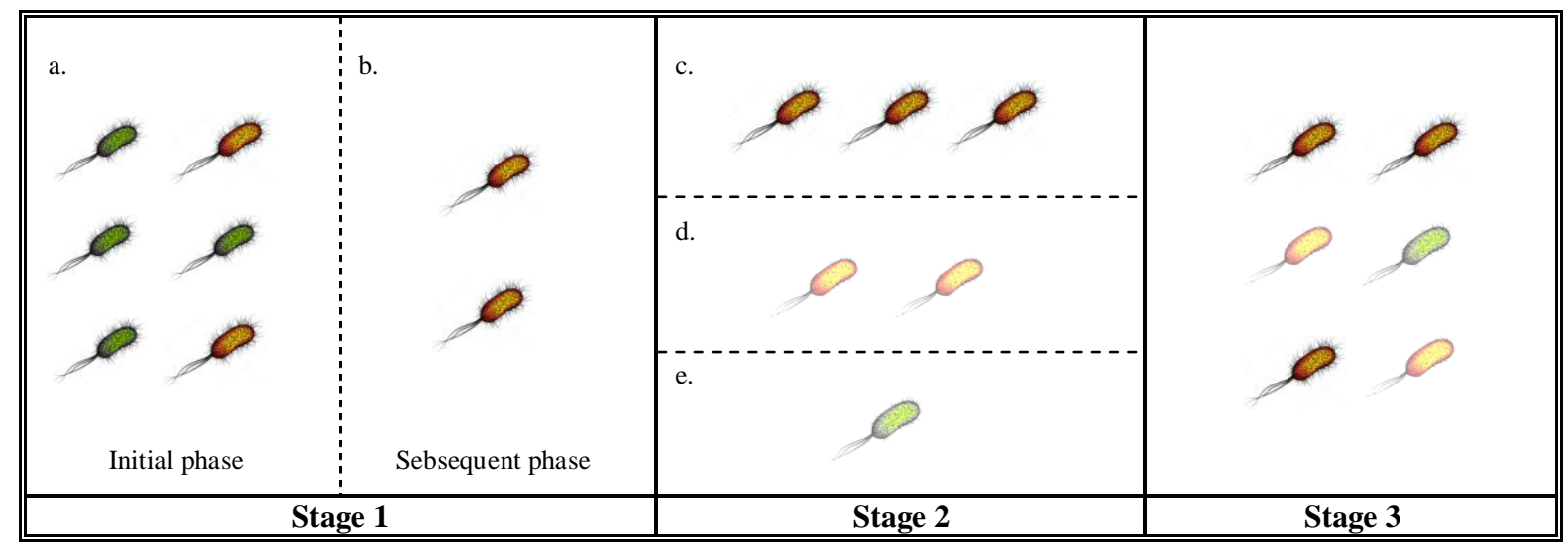

Figure 2. Evolutionary processes occurring in the stages of lithopanspermia. Stage 1: (a) the initial phase in which the bacterial population encounters the asteroidal or cometary impactor; (b) the subsequent phase, still within the planetary boundary, in which only the surviving pressure and heat shock tolerant bacteria remain. Stage 2: (c) pressure and heat shock tolerant bacteria continue reproduction and natural selection of robust genotypes. (d) pressure and heat shock tolerant bacteria undergo phenotypic stress hardening and continuing reproduction and natural selection of robust genotypes. (e) pressure and heat shock tolerant bacteria enter a dormant state. Stage 3: the new pressure and heat shock tolerant bacterial population arrive. Notably, all three phases in stage 2 can occur simultaneously among individuals in the population, and (e) in stage 2 may also occur after the phases in (c) and (d), but before entering stage 3. Credits: bacteria adapted from Mirumur, 2011.

During stage 2 from the donator world to the acceptor world, the temperature inside the meteorite follows either the temperature in space or is higher because of an internal heat residue left over from stage $1-\mathrm{a}$ potential heat source originating elsewhere could also perhaps be conceivable.

Thus, in principle, a gradual transition could occur, wherein the bacteria initially have a life cycle within the meteorite in stage 2 but, as the meteorite gradually gives way to the pressure from space and the bacteria gradually run out of nutrients, switch to a dormant state that can protect them for the remainder of the transmission; see Figure 2, Stage 2 (e).

The few bacteria surviving stage 1 can be expected to potentially survive stage 3 , because they already possess inherent trait robustness. However, such a gradual transition may be important for reasons discussed below. If the bacteria are active for a period of time in stage 2 , then evolutionary processes will be in effect.

Bacteria have a life cycle much faster than those of multi-cellular organisms. The doubling times of different bacteria can vary considerably, from between 9.8 minutes to several hours [Eagon, 1962; Gibson et al., 2018]. Thus, if a bacterium in a meteorite is assumed to have a doubling time of 30 minutes, in just 24 hours, $2.81 \times 10^{14}$ bacteria could arise, potentially comprising 48 generations. Therefore, in principle, the meteorite must protect the bacteria only a few hours in stage 2 to allow them to undergo multiple rounds of reproduction and natural selection and consequently evolution.

Moreover, as a side note, viable halophilic microorganisms are well known to be consistently cultivated from fluid inclusions in halite, where they may have been trapped from decades to perhaps millions of years (for review, see e.g., Jakkola et al., 2016). McGenity et al. (2008) have calculated that adequate nutrient resources might be present if haloarchaea were packed inside naturally forming halite crystals, such as a cubic fluid inclusions with dimensions of $100 \mu \mathrm{m}$, able to contain 50,000 of these microorganisms. These cells could provide sustenance for a single haloarchaeon, functioning for the most part anaerobically, during 
a 100-million-year period by consuming this co-deposited organic matter of dead cells, which would provide sufficient carbon and energy [McGenity et al., 2008]. This situation may be different from that discussed here in stage 2, but it shows that such organisms can in principle survive extremely long periods under harsh conditions with limited internal resources.

However, despite these considerations, ad oculos, the premise of lithopanspermia is that organisms can be transferred safely to another solar system body. If, for the sake of discussion, we generously assume that travel time is short and/or that a meteorite protects the bacterial population to such an extent that the bacteria not only remain alive but also remain active in stage 2 throughout the transmission, then the bacteria will be able to show this increased robustness. But then another scenario emerges, because then, as described, evolutionary processes will be in effect.

In the previous scenario, stage 1 and stage 3, there would be high mortality in the bacterial population through stage 1, after which the surviving individuals would later experience a second stress event of the same type as that in stage 3. However, the number of bacteria would not increase again throughout the stages of lithopanspermia.

However, if the meteorite through stage 2 ensures that the surviving bacteria are not only able to maintain the most basic functions but also to undergo reproduction and natural selection, then a related but new scenario arises.

First, we have the situation in which a physiological state may be conferred upon the surviving bacteria that will increases robustness towards a second stress challenge of the same type in stage 3. Second, we also have the situation in which an increase in quantity or population size occurs, because the bacterial population is able to remain active with reproduction and selection. Here, the potential increase in quality will thus be twofold. The frequency increase in robustness will occur as in the first scenario, thereby increasing the quality of the robustness of the bacteria. Furthermore, an increase in the quantity of bacteria will also occur, thereby enhancing the inherent trait of robust genotypes that evolved on the donator world, and distributing this quality among the growing population as well.

Thus, not only a few survivors but a large population of bacteria would potentially display enhanced tolerance to stress after arriving in an acceptor world, exhibiting both this robustness and the inherent robustness to stress. Hence, a potentially large group of bacteria would be able to cope with the stressors in stage 3 , through possessing a greater mean tolerance to the same type of stress than would be expected from a control group that did experience a stress event in stage 1.

Another scenario can be described. If the bacteria in stage 2 were protected but still experienced environmental stressors more severe than those in their native environment in the donator world, then they could not only undergo evolutionary adaptation and become better at coping with the environment existing in stage 2 in the meteorite ${ }^{1}$ but also undergo stress hardening; see Figure 2, stage 2, (d).

In stage 1, it was the pre-adapted heat stress robust bacteria that survived. In stage 2 heat stress may be experienced during several processing steps rather than all at once, because time may be available, thus potentially leading to stress-induced protection of the bacteria. Stress hardening is part of a phenotypic response that an organism may have towards environmental stress [Lou and Yousef, 1996]; therefore, if the first encountered stress inside the meteorite is not fatal to the bacteria, the adaptation of the bacteria towards this subfatal stress may increase their robustness and allow for their survival under subsequent stresses. Mackey and Derrick (1986) have shown that such heat resistance can emerge rapidly before the subsequent stress interval, reaching virtually maximum resistance within only $30 \mathrm{~min}$.

Of course, if stress hardening occurs among some of the bacteria in the donator world environment, where the asteroidal or cometary impactor landed and ejected them in stage 1, then they would thus have both a genotypic adaptation and a phenotypic response that could increase their survival probability.

\footnotetext{
${ }^{1}$ Of course, if they can remain active in the environment of the meteorite, and the transmission lasts long enough, then the bacterial population can eventually evolve traits that remove robust genotypes towards pressure and heat shock, and instead confer genotypes robust towards other stressors. Here, for simplicity's sake, they are assumed to maintain their robust genotypes towards pressure and heat shock.
} 
Stress hardening might be considered the reason why the bacterial population is conferred this state that mitigates the effects of the second stress event of the same type. However, the results of Lenz et al. (2018) have indicated that it was in fact two different mechanisms due to the recovery time.

Although increased heat tolerance in the bacterial population is a temporary state (Rockabrand et al., 1995), this reversible phenotypic response still confers stress tolerance on an organism that can persist even after stress in the meteorite environment again disappears, owing to the persistence of the heat stress proteins for brief time spans in the stress hardened bacteria, as well as to the synthesis of the characteristic set of stress proteins not terminating immediately after the stress inside the meteorite environment ceases; such synthesis may continue for hours, depending on the species [Bahrndorff et al., 2009].

Furthermore, adaptation to heat stress can also endow the bacterial population with protection against some of the other stresses that the population may encounter in stage 2, such as cold stress tolerance [Ferrer et al., 2003], which may be increasingly important protections in the environment of the meteorite.

Thus, the quantity of the bacterial population could greatly decrease through stage 1, but the quantity could potentially increase again through stage 2 (distributing quality along the way in the growing population); consequently, the quality of the bacterial population would increase before entering stage 3 . Hence, the evolutionary strategy would ensure an increase in quantity and thus quality in the meteorite. Thus, the first and second stages of lithopanspermia can themselves be considered facilitators of an evolutionary process that enhances the ability of the collected bacteria and the possibility of surviving the transmission to an acceptor world.

\section{The stages of lithopanspermia and invasiveness}

The stages of lithopanspermia are not only important vectors that allow a population of bacteria to reach a new solar system body. These stages can also be assumed to increase the potential of the bacteria to invade the new environment in the acceptor world by enhancing the bacterial population's tolerance to adverse environmental conditions.

Thus, e.g., heat shock can potentially induce changes that protect bacteria from not only thermal stress but also other stress conditions. Hence, the bacteria that obtain tolerance towards a specific stressor within the meteorite can in fact also achieve enhanced protection against other subsequent stresses that may be encountered, a phenomenon referred to as cross-protection [Johnson, 2002]. Thus, such stress-hardened organisms synthesize a characteristic set of stress proteins [VanBogelen et al., 1987], which allow the bacterial population to obtain protection against other stresses. Several stresses beyond heat stress have been demonstrated to induce cross-protection, such as cold stress [Periago et al., 2002], osmotic stress [Mattick et al., 2000], starvation stress, and even ultraviolet radiation stress [VanBogelen et al., 1987; Watson, 1990].

Thus, the multiple abiotic pressures that the bacterial population encounters within the meteorite in stage 2 can potentially lead to development of very robust bacteria with highly enhanced survival capacities. Hence, even though the environment of the donator world and the acceptor world, and the environment of the meteorite and acceptor world may be very different, although both are habitable, this enhancement of survival capabilities could still be expected to enhance invasiveness in a potential founder population such as the arriving bacterial population.

However, as indicated by von Hegner (2020), reaching another world does not mean that life can survive its encounter with this world. In encountering the environment in a new world, an all-or-nothing response is in effect, and bacteria must survive the first encounter.

As described, a time lag in adaptation are unavoidable as populations experience environmental change [Simons, 2011]. Such time lags can be a major obstacle to survival of life encountering another world, because although organisms arrive in an environment in the acceptor world that is potentially life friendly, they do not have time to adapt if the environment is too different from the original environment in the donator world. Thus, the new environment must either be similar to the environment to which they are adapted — a probability that is not high — or the organisms must use an evolutionary mode of response called bet hedging.

Bet hedging is an evolutionary strategy that generates stochastic variation in fitness-related traits among terrestrial bacteria, essentially distributing risk among an array of phenotypes, each of which is neither optimal nor a failure across multiple environments. This strategy increases the probability that some of these 
arriving bacteria will have a phenotype ensuring their immediate survival in this new unpredictable world [von Hegner, 2020].

However, assuming that the meteorite places the bacteria in a similar environment to that of the donator world or the environment to which they have adapted to through stage 2, then the enhanced tolerance towards different types of environmental stressors the bacteria achieved on the transmission will indeed be relevant during the initial stages of invasion, when the bacteria encounter a similar environment that may nevertheless present them with varying degrees of harsh abiotic conditions.

One additional factor should be mentioned. Stage 1 eliminated all the bacteria unable to cope with the pressure and heat shock. Thus, as described, although the few surviving bacteria could not be active through stage 2 and give rise to an increased population, the individual bacteria that survived stage 1 would clearly still have the potential to cope with stage 3 . However, this scenario would include only a few bacteria arriving. However, according to invasion biology, when expanding into new habitats, invading organisms are well known to rarely get a foothold in the first attempt. In many cases, these first invading organisms do not survive in the new environment. Several attempts are usually required, through a phenomenon called propagule pressure, before organisms become established. Hence, large or consistent releases of individuals into one new environment are necessary to enable survival establishment in the new environment [Lockwood et al., 2005].

In a terrestrial scenario, this aspect probably does not affect bacteria as much as multi-cellular organisms, owing to bacterial robustness and versatility. However, in an extra-terrestrial scenario, this aspect can matter. Thus, the survival of bacteria upon arrival in a new world will depend on the evolutionary strategy. If they arrive in a similar environment to that of the donator world, then the number of bacteria must be increased in the evolutionary process discussed to increase the probability of survival.

If they arrive in a life-friendly but still different environment from that of their donator world, then, as discussed by von Hegner (2020), the bacteria must use bet hedging to counter act several different environments at once. These strategies are not necessarily mutually exclusive, because bet hedging may exist in both scenarios; in fact, bet hedging may evolve on the transmission itself as a result of unpredictable environmental variance in the meteorite.

\section{Spacecraft mediated panspermia}

The previously discussed scenario belongs to a potentially possible, but probably modest, number of transmissions between worlds, because of dependence on the bacteria being able to remain active at least part of the time in stage 2. It cannot be excluded a priori that the meteorite will not be able to provide a protected environment where the bacterial population may be active. However, lithopanspermia is not the only possible relevant scenario. Beyond lithopanspermia, there is also a potential for spacecraft mediated panspermia.

This insight initially launched the discipline of exobiology, in which Lederberg (1960) clarified that organisms could potentially be transported by spacecrafts to other worlds. Planetary protection guidelines have been formulated since then (e.g., COSPAR Planetary Protection Policy, 2011), and the ethical implications of placing terrestrial organisms in other worlds have been considered, in a field closer to astrophilosophy than astrobiology; see von Hegner (2019).

With spacecraft mediated panspermia, stress-induced mortality that enhances the bacterial population's tolerance towards a second stress challenge of the same type is likely to be facilitated by a spacecraft. The analogue stages in a spacecraft mediated panspermia are not as violent in terms of pressure and heat shock as those in lithopanspermia. Still, the evolutionary processes will be in effect.

Thus, because a spacecraft has a significantly shorter travel time than most meteorites, and the interior of the spacecraft can be presumed to provide some protection to organisms and to provide a heat source as well, then the transmission between two solar system bodies is more likely to enhance the stress tolerance of hitchhiking organisms, thus potentially increasing their chances of survival in the new world. An opposite scenario of back contamination also exists in principle, wherein hypothetical life in other worlds unintentionally is transported back to Earth in spacecrafts, such that the robustness of alien organisms and their potential for invasiveness increases along the way. Thus, if a modern version of the Sagan-Coleman 
equation [Sagan and Coleman, 1965] were to be used, then it would need to include these evolutionary processes.

Forward contamination can be a more specific scenario. As indicated by von Hegner (2020), that life reaches another world does not necessarily mean that life can survive the encounter. In encountering the environment in a new world, an all-or-nothing response is in effect, wherein organisms must survive the first encounter.

The probability of surviving in the new environment at the first encounter is not high unless the environment matches the original environment. However, in spacecraft mediated panspermia, in missions that seek life elsewhere, e.g., an automated mobile laboratory, the situation are that this spacecraft are sent to locations on another solar system body, where the potential for life as we know it is suspected. Thus, we hypothetically can not only bring terrestrial organisms to a place where they are potentially more likely to survive but also increase their stress tolerance and cross-protection towards this new environment. Consequently, even if these organisms in the donator world would not be able to cope with such an environment, they can do so in the acceptor world, owing to this increase in survival capacities.

Thus, protocols that target what corresponds to stage 2 in lithopanspermia should be implemented, and if the specific and potentially habitable extra-terrestrial environment in which the spacecraft will arrive at is known, then all the mechanisms that potentially could enhance the capacity of a specific species to tolerate adverse conditions during transmission and settlement should be clarified so that they can be targeted.

\section{Conclusion}

The stages in lithopanspermia can themselves facilitate an evolutionary process that enhances the ability of the collected organisms to survive, by adapting them to be more robust after arrival in an acceptor world than they were in the donator world. The arriving bacterial population as a whole may thus actually have a greater tolerance to pressure and heat shock when arriving in the acceptor world than when ejected from the donator world, owing to this process. Thus, although it is known what a given population of specific bacteria can manage at the onset of a particular stage of lithopanspermia, the evolutionary response will change this robustness, thus providing insight that should be considered in estimating the survival probability of a population of organisms arriving in a new world.

In summary, an analysis to understand conditions occurring in a bacterial population being transported in the three stages of lithopanspermia between two solar system bodies was introduced. The analysis detailed a probably rare but possible mechanism in lithopanspermia, thus bridging evolutionary adaptation, planetary science, and invasive range expansion. The results of the analysis highlight the demands and restrictions that planetary protection protocols must implement because of the natural evolutionary mechanisms at play in the potential spacecraft mediated panspermia to a new planetary environment.

More work is needed. Applying evolutionary theory to the interplanetary exchange in life is a novel pursuit. However, the generality of this framework makes it a valuable starting point for analyses and modelling of ecological scenarios regarding transmissions of life, natural or artificial, between worlds in a solar system.

\section{References}

Artsutanov, Yuri; V Kosmos na Electrovoze; Komsomolskaya Pravda, July 31, 1960.

Bahrndorff S, Marien J, Loeschke V, Ellers J (2009) Dynamics of heat-induced thermal stress resistance and Hsp70 expression in the springtail, Orchesella cincta. Funct Ecol 23:233-239.

Beech, Martin, Coulson, Ian M. Comte, Mark: Lithopanspermia - The Terrestrial Input During the Past 550 Million Years, American Journal of Astronomy and Astrophysics 2018; 6(3): 81-90.

Brennecka, G. A. L. E. Borg, M. Wadhwa. Insights into the Martian mantle: The age and isotopics of the meteorite fall Tissint, Meteoritics \& Planetary Science. Volume 49, Issue 3; March 2014; Pages 412-418.

COSPAR Planetary Protection Policy, 20 October 2002; As Amended to 24 March 2011.

de Montlivault, Le Comte Eleonore-Jacques-Francois: Conjectures sur la réunion de la lune à la terre, et des satellites en général à leur planète principale... par un ancien officier de marine Reliure inconnue, Paris, 1821. 
Eagon,R. G. Psedomonas natrigens, a marine bacterium with a generation time of less than 10 minutes, J Bacteriol. 1962 Apr; 83(4): 736-737.

Ferrer, M.; Chernikova, T.N.; Yakimov, M.M.; Golyshin, P.N.; Timmis, KN. Chaperonins govern growth of Escherichia coli at low temperatures. Nat. Biotechnol. 2003, 21, 1266-1267.

Gavan, Elisa, Govers, Sander K., Michiels, Chris W. and Aertsen, Abram: Severely Heat Injured Survivors of E. coli O157:H7 ATCC 43888 Display Variable and Heterogeneous Stress Resistance Behavior, Front. Microbiol., 2016.

Gibson, Beth, Wilson, Daniel J., Feil, Edward and Eyre-Walker, Adam: The distribution of bacterial doubling times in the wild, Proc Biol Sci. 2018 Jun 13; 285(1880): 20180789.

Horneck, Gerda, Stöffler, Dieter, Ott, Sieglinde, Hornemann, Ulrich. Cockell, Charles, S Möller, Ralf, Meyer, Cornelia, de Vera, Jean-Pierre Fritz, Jörg Schade, Sara and Artemieva Natalia A., Microbial Rock Inhabitants Survive Hypervelocity Impacts on Mars-Like Host Planets: First Phase of Lithopanspermia Experimentally Tested, Astrobiology, Vol. 8, No. 1, 2008.

Irving, Tony: http//www.imca.cc/mars/martian-meteorites-list.htm.

Jaakkola, Salla T., Ravantti, Janne, Oksanen, Hanna M., Bamford, Dennis H. Buried Alive: Microbes from Ancient Halite, Trends in Microbiology, Review, Volume 24, Issue 2, P148-160, 2016.

Johnson, E.A. Chapter 4: Microbial Adaptation and Survival in Foods. In Microbial Stress Adaptation and Food Safety; Yousef, A.E., Juneja, V.K., Eds.; CRC Press: Boca Raton, FL, USA, 2002.

Lederberg, Joshua. 1960a. "Exobiology: Experimental Approaches to Life Beyond the Earth." H.K. Kallmann Bijl (ed.), Space Research: Proceedings of the First International Space Science Symposium. Amsterdam: North Holland, pp. 1153-1170.

Lenz, Mark, Ahmed, Yasser, Canning-Clode, João, Diaz, Eliecer, Eichhorn, Sandra, Fabritzek, Armin G., da Gama, Bernardo A. P., Garcia, Marie, von Juterzenka, Karen, Kraufvelin, Patrik, Machura, Susanne, Oberschelp, Lisa, Paiva, Filipa, Penna, Miguel A., Ribeiro, Felipe V., Thiel, Martin, Wohlgemuth, Daniel, Zamani, Neviaty P., Wahl, Martin: Heat challenges can enhance population tolerance to thermal stress in mussels: a potential mechanism by which ship transport can increase species invasiveness, 2018 Nature.

Lockwood JL, Cassey P, Blackburn T (2005) The role of propagule pressure in explaining species invasions. Trends in Ecology and Evolution, 20, 5, 223-228.

Lou, Y., and A. E. Yousef. 1996. Resistance of Listeria monocytogenes to heat after adaptation to environmental stresses. J. Food Prot. 59:465-471.

Mackey, B.M. and Derrick, C.M. (1986) "Elevation of the heat resistance of Salmonella typhimurium by sublethal heat shock'. J. Appl. Bacteriol. 61,389-93.

Mattick, K.L.; Jorgensen, F.; Legan, J.D.; Lappin-Scott, H.M.; Humphrey, T.J. Habituation of Salmonella spp. at Reduced Water Activity and Its Effect on Heat Tolerance. Appl. Environ. Microbiol. 2000, 66, 49214925.

McGenity, T.J., Hallsworth, J.E. and Timmis, K.N. (2008) Connectivity between 'ancient' and 'modern' hypersaline environments, and the salinity limits of life. CIESM Workshop Monographs no.33: The Messinian Salinity Crisis from Mega-deposits to Microbiology - A Consensus Report. Almeria (Spain), $7-$ 10 November 2007, pp. 115-120.

Melosh, H.J. (1988) The rocky road to panspermia. Nature 332, 687-688.

Mileikowsky, C., Cucinotta, F.A., Wilson, J.W., Gladman, B., Horneck, G., Lindegren,L. , Melosh, J., Rickman, H., Valtonen, M., and Zheng, J.Q. (2000) Natural transfer of microbes in space; 1. From Mars to Earth and Earth to Mars. Icarus, 145(2): 391-427.

Nicholson, WL: Ancient micronauts: interplanetary transport of microbes by cosmic impacts. Trends Microbiol, 2009 Jun;17(6):243-50.

Nicholson, WL, Munakarta, N, Horneck, G, Melosh, HJ, Setlow, P. Resistance of Bacillus endospores to extreme terrestrial and extraterrestrial environments. Microbial Mol Biol Rev. 2000 Sep;64(3):548-72.

Nyquist, L.E., Bogard, D.D., Shih, C.-Y., Greshake, A., Stöffler, D., Eugster, O., 2001. Ages and geologic histories of martian meteorites. In: Kallenbach, R., Geiss, J., Hartmann, W.K. (Eds.), Chronology and Evolution of Mars. Kluwer Academic Publishers, Dordrecht, pp. 105-164.

Periago, P.M.; Schaik, W.V.; Abee, T.; Wouters, J.A. Identification of Proteins Involved in the Heat Stress Response of Bacillus cereus ATCC 14579. Appl. Environ. Microbiol. 2002, 68, 3486- 3495.

Richter, H. (1865) Zur Darwinschen Lehre. Schmidts Jahrb. Ges Med 126:243-249. 
Rockabrand, D. Arthur, T, Korinek, G, Livers, K and Blum, P. An essential role for the Escherichia coli DnaK protein in starvation-induced thermotolerance, $\mathrm{H} 2 \mathrm{O} 2$ resistance, and reductive division. J Bacteriol. 1995 Jul; 177(13): 3695-3703.

Sagan, C., Coleman, S. (1965). "Spacecraft sterilization standards and contamination of Mars". Journal of Astronautics and Aeronautics 3 (5): 22-27.

Sakai AK, Allendorf FW, Holt JS, Lodge DM, Molofsky J, With KA, Baughman S, Cabin RJ, Cohen JE, Ellstrand NC, McCauley DE, O’Neil P, Parker IM, Thompson JN, Weller SG (2001) The population biology of invasive species. Annu Rev Ecol Syst 32:305-332.

Simons, Andrew M. Modes of response to the environmental change and the elusive empirical evidence for bet hedging, Proc Biol Sci. 2011 Jun 7;278(1712).

The Meteoritical Society, 2020. http://www.lpi.usra.edu/meteor/metbull.php.

VanBogelen, R. A., P. M. Kelley, and F. C. Neidhardt. 1987. Differential induction of heat shock, SOS, and oxidation stress regulons and the accumulation of nucleotides in Escherichia coli. J. Bacteriol. 169:26-32.

von Hegner, Ian: Astrobiology and Astrophilosophy: Subsuming or bifurcating disciplines? Philosophy and Cosmology, vol. 23, 2019.

von Hegner, Ian: Interplanetary transmissions of life in an evolutionary context, International Journal of Astrobiology, 2020.

Watson, K. 1990. Microbial stress proteins. Adv. Microb. Physiol. 31:184-223. 